\title{
The Adjustment of Texture Mapping based on Image-Entropy
}

\author{
XiqingZhao ${ }^{1, ~ a}$, WenyingZhang ${ }^{1,}$, PengSun $^{1, a}$, LijunWang $^{1, \text { a }}$ \\ ${ }^{1}$ Hebei North University,Zhangjiakou,HeBei,China,075000 \\ aE-mail:zxqlytqq@163.com
}

Keywords: Block matching; image entropy; texture mapping.

\begin{abstract}
Mass terrain in four fork tree index structure Xia, draws Shi needs for effective of texture map, viewpoint of moved and texture retrieved Shi scene dynamic update directly effect to displayed efficiency, used macro points block match micro-texture map of method, effective to reduced has data of frequency refresh; introduced image entropy reflect image color distribution of space features, to improve original terrain texture, to out a more meet actual of terrain draws method; experimental indicates that in mass terrain texture map process in the, Increases the level of authenticity and real-time terrain rendering
\end{abstract}

\section{Introduction}

Large scale terrain texture data scenes display more lifelike, more granular, relying solely on GPU accelerated rendering technology, does not meet the requirements of practical application to draw realistic, lifelike effect of large-scale terrain environment, the need to use texture mapping to increase in entropy terrain of realism.

Since 1974, Catmull texture mapping method was first proposed, the three-dimensional surface texture generation technology has been an important research topic in computer graphics, and greatly promote the application and development of realistic graphics technology. Generated from the texture of view, 3D surface texture generation mainly three techniques, which Include Procedural texture synthesis, Texture mapping, Kind of map-based texture synthesis[1].

Wagner put forward a kind of after multiple texture sample plot Alpha fusion generation greatly static texture map method [2], this paper also discusses the method to achieve large-scale texture mapping through the hierarchy shiCache mechanisms.

In progressive mesh foundation that Hoppe in proposed, has realized GPU-based progressive mesh algorithm[3], he height map by the form memory of two-dimensional texture chart, in obviously saves, reduced the CPU load At present, for large scale terrain panoramic visualization wandering texture mapping processing is in a stage of study, has not formed a can be universally applicable method [4]. Although the domestic and overseas scholars put forward many different texture model, but these texture model fidelity is not enough, so large scale terrain rendering efficiency of static texture data organization in memory processing and real-time update combine to make the $3 \mathrm{~d}$ terrain simulation result has the sense of reality, this paper realize large-scale terrain based on the image entropy texture mapping panoramic roaming[5].

\section{Macro block matching}

Terrain image block. Topographical feature of the image such as color, texture, shape and spatial characteristics of the image is generally not distributed evenly in the entire image, after the block can quickly apparently unrelated block thrown away, and the rest go to extract characteristic.

Blocking policy provides for line-by-line and interlaced scan in two ways, usually interlace block classification actions in favor of image blocks. Of course, when there is a fast-moving, frame of spatial correlation between lines down, increase the spatial correlation between lines, field-based prediction can be used, on the contrary, when the movement has been very slow, with predictions based on frames. In addition, there are based on forecasts of dual forecast (Dual Prime), transmitted through a vector field and a small difference vector, requires more bits than the General to transfer 
motion vector based on some less predictable manner, thereby reducing the difficulty of judging, reducing a great memory, good for compressed data.

Terrain image block matching Map block after images, each image features can be very well used in a parameterized model characterization, it's called image matching, image is divided into several blocks, finding a motion vector for each block, and motion compensation prediction coding. Each frame macro block or blocks are based on previously encoded data to predict, forecast based on coded macro block, block and the value of the current macro blocks, block margin, the results are compressed transmitted to the decoder, the decoder need additional information such as (motion vector, forecasting models, and so on) is used to repeat the prediction process. Each divided region has its corresponding motion vector, and motion vectors and block approach to coding and transmission In more detail in the frame if you choose a large block size, means that is used to indicate motion vectors and the Division of regional types of bits would be less, but campaign compressed redundancy at more; if you select smaller block sizes, smaller campaign compressed redundancy, but require more bits than, It is necessary to weigh the influence of compression on the block size selection, General details for less, a relatively flat region Select a block size larger ,for more details in the image area select a block size smaller.

Micro-texture pretreatment. After more than block-matching, the actual terrain has been effectively converted, great original texture data or could not be read into memory at once ,So the need for sampling and segmentation the original data organization, namely for data preprocessing.

Samples are of low precision pixel data sampling a texture image. We use a simple average of the sampling methodologies are eight adjacent pixels to a new pixel values. A sampling process will get half of the texture image resolution. Repeat this action, we get a series of texture, uniform resampling pixel values of a set, it can create a textured pyramid model, starting from the initial 1,2,3 coding.

Segmentation refers to the texture textures cut into small blocks in the pyramid, its size was able to read the data into memory on time and not wasting system bandwidth. With index four fork tree coding to specified overall texture regional in the a specific of terrain block, by organization texture block save in name for block[-p][-q] of disk, block is original texture name, $p$ representative this texture block belonging to texture pyramid a a layer, q said in p layer of four fork tree node texture block, to formed double buffer structure to reduced disk to memory and memory to video of data exchange frequency.

Code reflects the textured area location of the entire texture, QuadTree coding can quickly get through the index of the current texture regional node, the father of coding. If the length of the current texture coding region QuadTree nodes is n, it's father region QuadTree encoding is that it n-l the first letter of a number of nodes.

Texture data processing. Each of texture Pyramid, only then minority texture block close viewpoint read in the memory. Along with the migration of viewpoint, must continuously renew from the floppy disk to the data of memory. But the read way solely depends upon the parent-child operator of quadtree unable to meet the need.

As the viewpoint of move, in-memory textures need to be updated in real time, but update frequency affect rendering. To address the problem, use double buffering. First texture layer of cache storage is read from disk, the texture size is larger than the size of the view; second buffer storage is the first cache and Windows the same size texture layer. therefore Viewpoint, and moved a short distance and does not cause the Windows over the first cache storage range of texture, especially in low resolution layer, We only need to be updated from the first second cache to cache data, Only when the bounds of the window when we read data from the disk. So most of the time, when view moves over time Windows will first of the texture cache, This will go a long way to avoid frequent exchange of data between disk and memory and improved drawing speed.

Do not use any macros for the figures and tables. (We will not be able to convert such papers into our system.)

Image entropy-based identification. Topographic maps as block, motion vectors are the necessary parameters of the model, must be coded to join bitstream. Because motion vectors are not independent of, often using differential coding to reduce rates. This means before the adjacent motion 
vector coding them as poor, only encode the differential parts. Entropy encoding used to encode the motion vector component can further elimination of motion vector statistic redundancy[6].

The image entropy treats the color of the image as a random event using to determine entropy value The image entropy expressed the bits mean value of image sets.

Define $I(A)=-\log p(A)$ (Where $p(A)$ is the probability of event $A$ )is the self-information of event A using to measure the information content when event A occurs Use The average of the self-information of $\mathrm{N}$ possible results of the random events:

$H=-\operatorname{sum}(p(i) / \log (P(i)))$

called entropy( ,separately is the probability that $n$ events correspond)as a measure of the uncertainty of an event is the parameter that describes the disorder of things greater the entropy is stronger the disorder, the more uncertain events whose entropy is 0 are definite And P(i) represents the percentage.

Image entropy can represent characteristics of image color distribution and accumulation but can not reflect the image color space distribution characteristics therefore, introducing two-dimensional entropy of image Select neighborhood color values of the image as a color space distribution characteristics composes the feature two tuples with image's pixel color recorded as (i, j), which I represents pixel color values $(0<=\mathrm{i}<=255)$, $\mathrm{j}$ represents the domain $\operatorname{color}(0<=\mathrm{j}<=255)$,

$$
P_{i j}=f(i, j) / N^{2}
$$

This can reflect a location on the color value of the pixel and its surrounding pixels integrated characteristics of color distribution where $f(i, j)$ is the frequency of occurrence of the feature two tuples $\mathrm{f}(\mathrm{i}, \mathrm{j}), \mathrm{N}$ is the scale of image defined the image discrete two-dimensional entropy as:

$$
\mathbf{H}=\sum_{i=0}^{255} p_{i j} \log p_{i j}
$$

According to the structure of the image two-dimensional entropy can reflect the image contains the premise of information outstandingly reflect the comprehensive features of the image pixel position color information and pixel neighborhood of color distribution Following is a topographic images before and after the Elimination of cracks on entropy technology such as Figure 1.Through the judgment distance from the viewpoint to the distance of node center from reducing and dividing the two directions ,ultimately, eliminate the effect of crack to achieve different levels of detail of the terrain between smooth seamless transitions[7].

Figure 1 Elimination of cracks before and after.

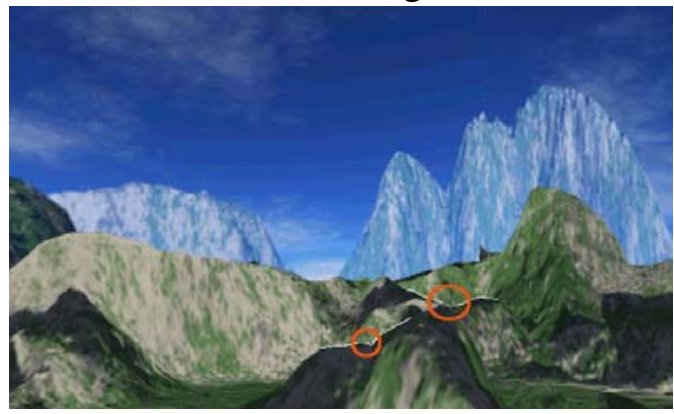

(a) Before the Elimination of cracks

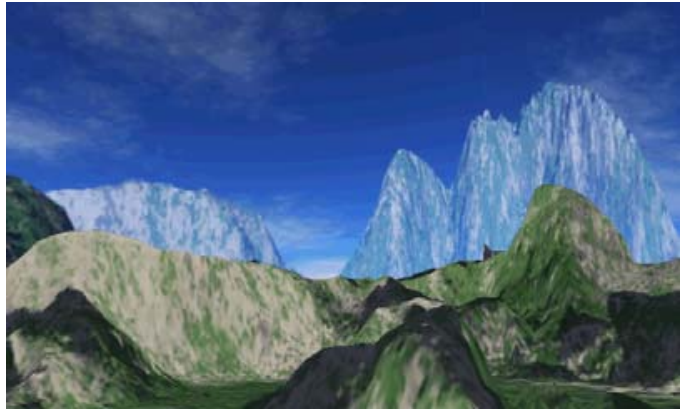

(b)After the Elimination of cracks

Analysis of the results. Contrast traditional Clipmap method and image-based entropy texture mapping adjustment technolog carries on the texture mapping technological experiment to terrain model of 1202x802 size under hierarchical structure the texture size is set to $10000 \times 6667,2$ bytes for each pixel the window size is $1024 \times 1024$ (pixels), The first cushion and second cushion that we established are both five layers storage space that requires is:512×512(textured pixel) $\times 5$ (layers) $+1024 \times 1024$ (textured pixel) $\times 5=12$.5MB,requires less space than Clipmap (as shown in table 1).The experimental result is the time of data exchange each time approximately is $40 \mathrm{~ms}$ between the hard disk and memory ,memory update time alone is approximately 28ms.As shown in table 2 , the average rate is about $46 f p s$. 


\begin{tabular}{|c|c|c|c|c|c|}
\hline Method & Clipmap & \multicolumn{4}{|c|}{ method of this article } \\
\hline & & \multicolumn{2}{|c|}{ The first cushion } & \multicolumn{2}{|c|}{ The second cushion } \\
\hline & & \multicolumn{2}{|c|}{$10 \mathrm{M}$} & \multicolumn{2}{|c|}{$2.5 \mathrm{M}$} \\
\hline $\begin{array}{l}\text { Total } \\
\text { Space }\end{array}$ & $36.5 \mathrm{M}$ & \multicolumn{4}{|c|}{$12.5 \mathrm{M}$} \\
\hline \multicolumn{6}{|c|}{ Table 2 this method view frame rate } \\
\hline \multicolumn{2}{|c|}{ Window size } & Maximum & \multicolumn{2}{|c|}{ Minimum } & Average \\
\hline \multicolumn{2}{|c|}{$512 \times 512$ (pixel) } & 53fps & \multicolumn{2}{|c|}{ 39fps } & 46fps \\
\hline
\end{tabular}

\section{Summary}

Under the structure of QuadTree indexing The method of macroscopic image blocking matching micro texture mapping effectively reduces the frequency of data refresh introduces the image entropy to reflect the space characteristics of image color distribution improves the original terrain textures and gives a more realistic terrain rendering method satisfies the timeliness of large-scale terrain panoramic roaming makes terrain rendering frame rate improved greatly.

\section{References}

[1] Catmull. A class of local interpolating splines. Comuter Aided Geometric Design, R, E. Barbhill and R. F. Reisenfeld, Eds. Academic Press, New York, 1974:317-326

[2] Wagner,D. 2004.Terrain geomorphing in the vertex shader. In ShaderXZ:Shader Programming Tips\& Tricks with DirectX9 .Word ware Publishing

[3] Asirvatham A, Hoppe H. Terrain Rendering Using GPU-Based Geometry Clipmaps. GPU Gem: 27-45.

[4] Williams, Lance. Pyramidal Parametrics. In Peter Tanner, editor, Computer Graphics (SIGGRAPH 83 Conference Proceedings). ACM SIGGRAPH, 1983:1-11

[5] Tanner, Migdal. The Clipmap: A Virtual Mipmap. In Proceedings of SIGGRAPH, 1998:151-159

[6] Jonathan Blow. Terrain Rendering at High Level of Detail. Paper for the Game Developers' Conference. 2000

[7] Wei Hua, Huaisheng Zhang, Yanqing Lu, Hujun Bao, Qunsheng Peng. Huge Texture Mapping for Real-Time Visualization of Large-Scale Terrain. ACM SIGGRAPH, 2004:154-157 